\title{
Memory and Chaos Effects in Spin Glasses
}

\author{
K. Jonason ${ }^{2}$, E. Vincent ${ }^{1}$, J. Hammann ${ }^{1}$, J.P. Bouchaud ${ }^{1}$, P. Nordblad ${ }^{2}$ \\ ${ }^{1}$ Service de Physique de l'Etat Condensé, CEA Saclay, \\ 91191 Gif sur Yvette Cedex, France \\ ${ }^{2}$ Department of Material Science, Uppsala University, P.O. Box 534, \\ 75121 Uppsala, Sweden
}

\begin{abstract}
New low frequency ac susceptibility measurements on two different spin glasses show that cooling/heating the sample at a constant rate yields an essentially reversible (but rate dependent) $\chi(T)$ curve; a downward relaxation of $\chi$ occurs during a temporary stop at constant temperature (ageing). Two main features of our results are: (i) when cooling is resumed after such a stop, $\chi$ goes back to the reversible curve (chaos) (ii) upon re-heating, $\chi$ perfectly traces the previous ageing history (memory). We discuss implications of our results for a real space (as opposed to phase space) picture of spin glasses.
\end{abstract}

PACS numbers: $75.50 . \operatorname{Lk} 75.10 . \mathrm{Nr}$

to appear in Phys. Rev. Lett.

The dynamic properties of the spin glass phase have been extensively studied by both experimentalists and theorists for almost two decades [1,2]. The observed properties reflect the out-of-equilibrium state of the system: the response to a field variation is logarithmically slow, and, in addition, depends on the time spent at low temperature ("ageing"). Ageing is fully reinitialized by heating the sample above the glass temperature $T_{g}$. It corresponds to the slow evolution of the system towards equilibrium, starting at the time of the quench below $T_{g}$. Many aspects of ageing are similar to the "physical ageing" phenomena that have been characterized in the mechanical properties of glassy polymers [3]. In the last few years, some interesting progress in the theoretical understanding of ageing in disordered systems has been achieved [4].

From the studies of the critical behaviour at $T_{g}$ [5], it appears that the approach of $T_{g}$ is associated to the divergence of a spin-spin correlation length, as is the case in the phase transition of classical ordered systems. In the spin glass phase, the system is out of equilibrium: as in simple ferromagnets, it is tempting to associate ageing with the progressive growth of a typical domain size towards an equilibrium infinite value. However, this simple picture cannot account for all the experimental observations. In particular, the effect of small temperature cycles (within the spin-glass phase) is rather remarkable [6.7]:

- on the one hand, ageing at a higher temperature barely contributes to ageing at a lower temperature. Said differently (as will be discussed again below), the thermal history at sufficiently higher temperatures is irrelevant. This is at variance with a simple scenario of thermal activation over barriers, where the time spent at higher temperature would obviously help the system to find its equilibrium state. Everything happens as if there were strong changes of the free-energy landscape with temperature. This point is suggestive of the "chaotic" aspect of the spin glass phase that has been predicted from mean field theory [8] and from scaling arguments in 9,10$]$.

- on the other hand, interesting memory effects concomitantly appear: the state reached by the system at a given temperature can be retrieved after a negative temperature cycle.

In the present letter, we describe some new experiments which reveal in a rather striking way these memory and chaos effects, and we point out their implications for the construction of a real space picture of spin glasses. The results are obtained using a new experimental protocol, which has first been proposed and applied to the metallic $\mathrm{Cu}: \mathrm{Mn}$ spin glass by one of us 11]. We now develop this approach in a series of measurements on the $C d C r_{1.7} \operatorname{In}_{0.3} S_{4}$ insulating spin glass 12]. The universality of the out-of-equilibrium dynamics in spin glasses is evidenced by the similarity between the results on two very different realisations of spin glass systems.

In this procedure, we record the ac-susceptibility of the sample as a function of temperature. The ac field has a low frequency of $\omega / 2 \pi=0.04 \mathrm{~Hz}$, to allow the relaxation of the susceptibility due to ageing to be clearly visible on the scale of several hours. The peak amplitude of the ac field is $0.3 O e$, which is low enough not to affect the properties of the system. We cool the system from above $T_{g}=16.7 \mathrm{~K}$ down to $5 \mathrm{~K}$ at a constant cooling rate of $0.1 \mathrm{~K} / \mathrm{min}$, and then heat it back continuously at the same rate. This yields two slightly different curves; the one obtained upon heating (a bit below the other one) is chosen as the reference curve, and shown as a solid line in Fig. 1. We then repeat the experiment, but now stop during cooling at an intermediate temperature $T_{1}=12 \mathrm{~K}$ during a certain waiting time $t_{w 1}=7 \mathrm{~h}$. During $t_{w 1}$, due to ageing, both $\chi^{\prime}$ and $\chi^{\prime \prime}$ relax downwards, by about the same amount; for $\chi^{\prime \prime}$, however, the relative amount is much larger, which makes the effect more visible, and in the following we mainly concentrate on the out-of-phase component. After the ageing stage at $T_{1}$, the cooling procedure resumes, and one observes 
that $\chi^{\prime \prime}$ and $\chi^{\prime}$ merge back with the reference curve only a few Kelvin below $T_{1}$. Thus, ageing at $T_{1}=12 \mathrm{~K}$ has not influenced the result at lower temperatures ( chaos" effect).

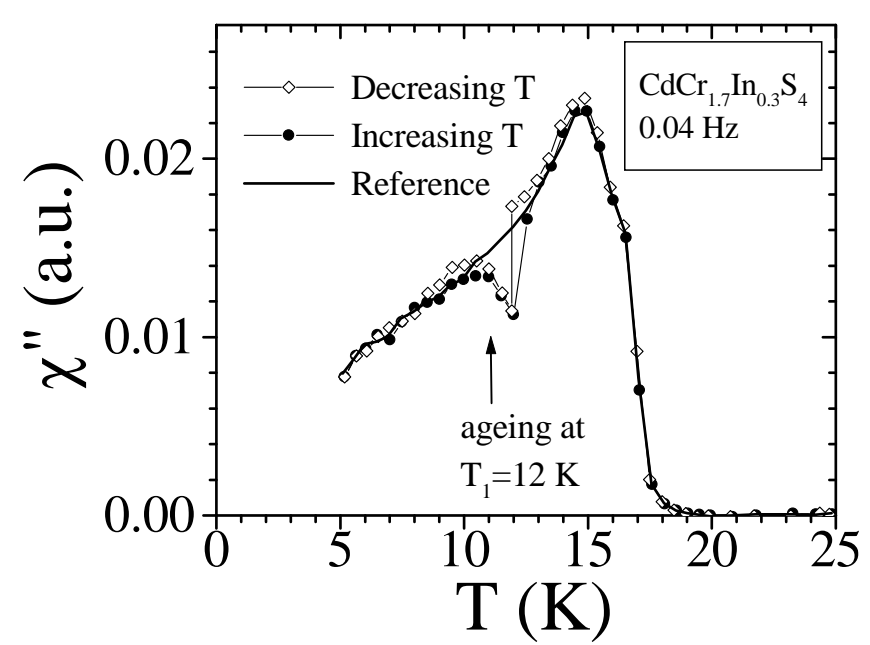

FIG. 1.

Out-of-phase susceptibility $\chi^{\prime \prime}$ of the $C d C r_{1.7} \operatorname{In}_{0.3} S_{4}$ spin glass. The solid line is measured upon heating the sample at a constant rate of $0.1 \mathrm{~K} / \mathrm{min}$ (reference curve). Open diamonds: the measurement is done during cooling at this same rate, except that the cooling procedure has been stopped at $12 K$ during $7 h$ to allow for ageing. Cooling then resumes down to $5 K: \chi^{\prime \prime}$ is not influenced and goes back to the reference curve ("chaos"). Full circles: after this cooling procedure, the data is taken while re-heating at the previous constant rate, exhibiting memory of the ageing stage at $12 K$.

The surprise is that when the sample is re-heated at a constant heating rate (i.e. no further stops on the way up), we find that the trace of the previous stop (the dip in $\chi^{\prime \prime}$ ) is exactly recovered (see Fig 1 ). The memory of what happened at $T_{1}=12 \mathrm{~K}$ has not been erased by the further cooling stage, even though $\chi^{\prime \prime}$ at lower temperatures lies on the reference curve. The system can actually retrieve information from several stops if they are sufficiently separated in temperature. In Fig.2, we show a "double memory experiment", in which two ageing evolutions, one at $T_{1}=12 \mathrm{~K}$ and the other at $T_{2}=9 \mathrm{~K}$, are retrieved [13]. In the inset of Fig.2, the result of a similar experiment on a $\mathrm{Cu}: \mathrm{Mn}$ sample is shown [11].

As discussed above, the cooling rate dependence of the dynamics in spin glasses is largely governed by the "chaos" effect. For example, it has been shown that there is no difference in the ageing behaviour if the spin glass has been directly quenched from above $T_{g}$ or if it has been subjected to a very long waiting pause immediately below $T_{g}$ [7]. However, the influence of the cooling rate was not quantitatively characterized in systematic measurements, and this point is of a particular interest for the compar- ison between spin glasses and other glassy systems. We have therefore performed the following experiment. We cool the sample progressively and continuously (in fact, by steps of $0.5 \mathrm{~K}$ ) from above $T_{g}$ to $12 \mathrm{~K}=0.72 T_{g}$, using three very different cooling rates. The result is shown in Fig.3. The initial values of $\chi^{\prime}$ and $\chi^{\prime \prime}$ are indeed different: slower cooling yields a smaller initial value of the susceptibility, a value that is closer to "equilibrium". A small horizontal shift of the curves along the time scale allows the superposition of the three of them; the curves obtained after a slower cooling are somewhat "older". However, all curves are clearly converging towards the same asymptotic value. This behaviour contrasts with that observed in systems where thermally activated domain growth is important, for example the dielectric relaxation of the dipole glass $\mathrm{K}_{1-x} \mathrm{Li}_{x} \mathrm{TaO}_{3}$ [14]. There, it is found that different cooling rates lead the system to very different apparent asymptotic values of the dielectric constant.

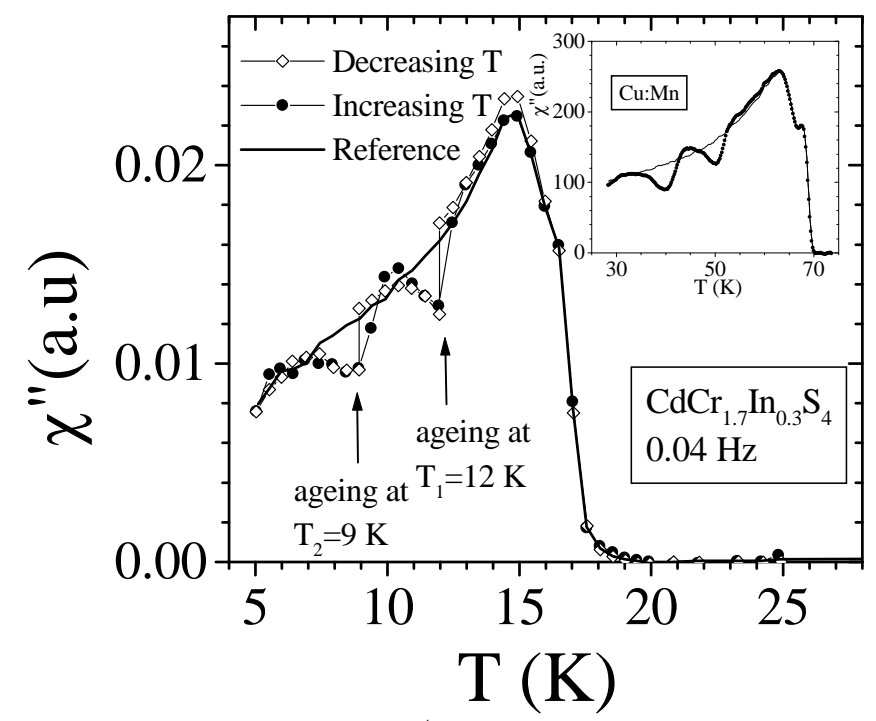

FIG. 2. Same as in Fig.1 ( $C d C r_{1.7} \operatorname{In}_{0.3} S_{4}$ insulating sample), but with two stops during cooling, which allow the spin glass to age $7 h$ at $12 K$ and then $40 h$ at $9 K$. Both ageing memories are retrieved independently when heating back (full circles). The inset shows a similar "double memory" experiment performed on the Cu:Mn metallic spin glass [11].

One can furthermore show that the cooling rate effect seen in Fig. 3 is entirely due to the last temperature interval, and not at all to the time spent at higher temperatures. We again use different cooling rates from above $T_{g}=16.7 \mathrm{~K}$ to $14 \mathrm{~K}$, but then we rapidly cool from $14 \mathrm{~K}$ to $12 K$, where the relaxation is measured. In this procedure, despite very different average cooling rates, the last two Kelvin are always crossed at the same speed. The result, in Fig. 3 (inset), is unambiguous: the obtained relaxation is the same in all cases, for $\chi^{\prime}$ as well 
as for $\chi^{\prime \prime}$. Thus, in a spin glass, the only influence of the cooling rate on the ageing state is due to the very last temperature interval before reaching the measurement temperature, while due to chaos effects the time spent at higher temperatures does not contribute. Again, this strongly contrasts with the case of $\mathrm{K}_{1-x} \mathrm{Li}_{x} \mathrm{TaO}_{3}$ alluded to above, where it is the time spent at temperatures near the glass transition that mostly determines the final state after the quench [14].

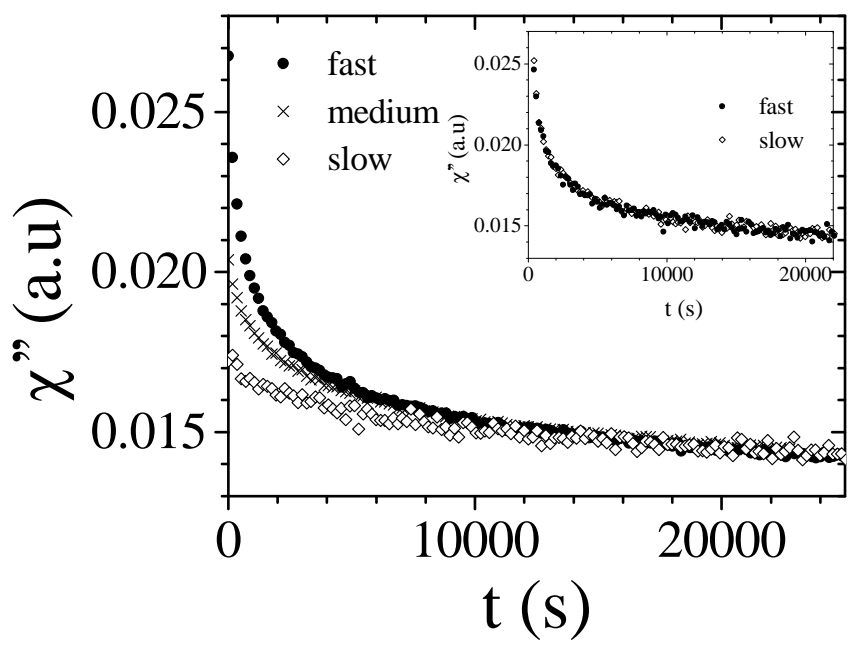

FIG. 3. $\chi^{\prime \prime}$ relaxation at $12 K$ as a function of time: effect of the cooling rate on ageing. The $C d C r_{1.7} I n_{0.3} S_{4}$ sample has been cooled from above $T_{g}=17 \mathrm{~K}$ to $12 \mathrm{~K}$ at very different speeds: $2.6 \mathrm{~K} / \mathrm{min}$ (full circles), $0.08 \mathrm{~K} / \mathrm{min}$ (crosses), $0.015 \mathrm{~K} / \mathrm{min}$ (open diamonds). In the inset, another procedure is used which shows that this cooling rate effect is only due to the last temperature interval: constant rate of $0.8 \mathrm{~K} / \mathrm{min}$ (full circles) or $0.08 \mathrm{~K} / \mathrm{min}$ (open diamonds) from $17 K$ to $14 K$, but in both cases rapid quench from $14 K$ to $12 K$.

There has been quite a number of approaches, inspired by Parisi's solution of mean-field models, in which ageing can be described in terms of a random walk in the space of the metastable states [15] 17]. The memory and chaos effects have been described in terms of a hierarchical organization of the metastable states as a function of temperature [7], a picture in which the growth of the free energy barriers when the temperature decreases could be characterized quantitatively [18]. Although these phase space pictures are very helpful (and have been used very early in the context of spin-glasses and glasses), it is obvious that they need to be linked with real space pictures, where the experimental signal can be attributed to certain clusters of spins which flip collectively. Macroscopically, ageing means that the system becomes "stiffer" with time, in the sense that the response to a field variation becomes slower and slower with increasing age. Slower response means larger free-energy barriers, and correspondingly a larger number of spins to be simultaneously flipped: one is thus naturally led to think in terms of growing "domains" (or "droplets") of correlated spins. The simplest picture based on this idea has been proposed by Fisher and Huse [19] and Koper and Hilhorst [20] in slightly different terms. It is based on the postulate that, at any given temperature below the spin-glass transition, there is only one phase (and its spin reversed counterpart) to be considered, much as in a standard ferromagnet. The difference is of course that all spins do not point in one direction, but arrange in a random (but fixed) way imposed by the interplay between the disordered nature of the interactions and the temperature. One can however by convention call one of the phases 'up' and the other one 'down'; again as in a ferromagnet, the typical domain size is expected to grow with time, albeit logarithmically slowly since domain walls are pinned by the disorder.

In principle, this picture should lead to very strong cooling rate effects, which are, as stated above, not those that are observed experimentally in spin glasses (see Fig. 3 ). However, if one assumes (as suggested by mean field and scaling arguments) chaos with temperature, in the sense that the phase growing at temperature $T_{1}$ is not at all the "correct" equilibrium phase for another temperature $T_{2}$, the cooling rate dependence can indeed be small since the time spent at a higher temperature does not bring the system any closer to equilibrium. This must be contrasted with random field like systems, where the equilibrium state is the same in the whole low temperature phase, and where cooling rate effects are strong.

The chaotic dependence of the phase with temperature allows one to argue why ageing is restarted when the temperature is lowered: the configuration reached after ageing at $T_{1}$ is, from the point of view of the ' $T_{2}$-phase', completely random. Correspondingly, new $T_{2}$-domains have to grow. The problem, however, comes from the observed memory effect: it shows that the $T_{2}$-domains must indeed grow somewhere, but without destroying the preexisting $T_{1}$-domains. The only possibility is that the $T_{2}$-domains do not nucleate everywhere, but only around certain favorable nucleation centers, coexisting with the previous backbone of $T_{1}$-domains. This is however in contradiction with the idea that, at any temperature, only one phase (and thus two types of domains) is enough to describe the dynamics of the system completely, since we already require the coexistence of two types of domains ( $T_{1}$ - and $T_{2}$-domains). The same argument shows that, at the first temperature $T_{1}$, the system must actually be in a mixture of all the different phases encountered between $T_{g}$ and $T_{1}$. Intuitively, it seems clear that if the system is so fragile to temperature changes, then by the same token it is hard to imagine that other nearby 'phases' will not nucleate simultaneously with the nominal phase. In other words, the question is whether, at long times, the 'defects' are only domain walls between two well identified phases (as in the coarsening/droplet picture), or whether these defects are more complicated 
(branched) objects.

We believe that the memory effect discussed above is incompatible with a picture of the out-of-equilibrium dynamics based on one type of domains only. Obviously, this does not exclude the possibility that the equilibrium phase is unique; from an experimental point of view, however, the question is not relevant, since the system is never in equilibrium. A similar conclusion has been suggested by 'second noise spectrum' experiments [21], and by recent out of equilibrium numerical simulations [22].

The construction of a consistent 'hierarchical droplet' picture appears completely open and beyond the scope of the present paper. A possibility (discussed in 16, 4]) is that correlations at a given temperature establish themselves progressively, but only over non compact (i.e. fractal) clusters of spins, which are large enough to be frozen at that temperature, but leave the surrounding sea of spins relatively free. As the temperature is lowered, smaller clusters begin to freeze (and thus provide a new aging signal), while larger clusters are completely blocked (thus leading to the memory effect). Let us note that the idea of "droplets within droplets" has previously been discussed in [23,24 without (to our knowledge) getting to the stage of a more quantitative model. The present experiments, which suggest the coexistence of many different time scales (and thus, presumably, many length scales) may force one to take the idea of fractal droplets more seriously.

Acknowledgements Financial support from the Swedish Natural Science Research Council (NFR) is acknowledged; one of us (KJ) wants to thank the Swedish Institute (SI) for a fellowship. We are grateful to F. Alberici, L.F. Cugliandolo, P. Doussineau, A. Levelut, M. Mézard, M. Ocio, G. Parisi for enlightening discussions, and to L. Le Pape for his technical support.

[1] "Spin Glasses and Random Fields", Series on Directions in Condensed Matter Physics Vol.12, A.P. Young Editor, World Scient. 1998.

[2] E. Vincent, J. Hammann and M. Ocio in Recent Progress in Random Magnets, ed. D.H. Ryan (World Scientific, Singapore, 1992) cond-mat/9607224).

[3] L.C.E. Struik, "Physical Aging in Amorphous Polymers and Other Materials", Elsevier Scient. Pub. Co., Amsterdam 1978.

[4] J.-P. Bouchaud, L.F. Cugliandolo, J. Kurchan and M. Mézard, in op. cit. 酒 pp.161-223, and ref. therein.
[5] see e.g. references in E. Vincent and J. Hammann, J. Phys. C: Solid State Phys. 20, 2659 (1987).

[6] P. Granberg, L. Lundgren and P. Nordblad, J. Magn. Magn. Mater. 92, 228 (1990); P. Granberg, L. Sandlund, P. Nordblad, P. Svedlindh and L. Lundgren, Phys. Rev. B 38, 7097 (1988).

[7] Ph. Refregier, E. Vincent, J. Hammann and M. Ocio, J. Phys. (France) 48, 1533 (1987); E. Vincent, J.P. Bouchaud, J. Hammann and F. Lefloch, Phil. Mag. B 71, 489 (1995).

[8] M. Mézard, G. Parisi and M.A. Virasoro, "Spin Glass Theory and Beyond", World Scient. Lect. Notes in Phys. Vol. 9 (1987).

[9] A.J. Bray and M.A. Moore, Phys. Rev. Lett. 58, 57 (1987).

[10] D.S. Fisher and D.A. Huse, Phys. Rev. Lett. 56, 1601 (1986).

[11] P. Nordblad, unpublished; P. Nordblad and P. Svedlindh, in op. cit. [1] pp.1-27.

[12] M. Alba, J. Hammann, M. Ocio and Ph. Refregier, J. Appl. Phys. 61, 3683 (1987).

[13] Note that if $T_{2}$ is very close to $T_{1}$, ageing at $T_{2}$ slowly wipes out the memory of the dip at $T_{1}$. On the other hand, both dips are erased by heating above $T_{1}$.

[14] F. Alberici, P. Doussineau and A. Levelut, Europhys. Lett. 39, 329 (1997); F. Alberici-Kious, J.P. Bouchaud, L.F. Cugliandolo, P. Doussineau and A. Levelut, Aging in $\mathrm{K}_{1-x} \mathrm{Li}_{x} \mathrm{TaO}_{3}$ : a domain growth interpretation, condmat/9805208.

[15] K.H. Hoffmann and P. Sibani, Z. Phys. B Cond. Matt. 80, 429 (1990); K.H. Hoffmann, S. Schubert and P. Sibani, Europhys. Lett. 38613 (1997).

[16] J.-P. Bouchaud and D.S. Dean, J. Phys. I France 5, 265 (1995)

[17] Y.G. Joh, R. Orbach and J. Hammann, Phys. Rev. Lett. 77, 4648 (1996).

[18] J. Hammann, M. Lederman, M. Ocio, R. Orbach and E. Vincent, Physica A 185, 278 (1992).

[19] D.S. Fisher and D.A. Huse, Phys. Rev. B 38, 373 and 386 (1988).

[20] G.J.M. Koper and H.J. Hilhorst, J. Phys. France 49, 429 (1988).

[21] M.B. Weissman, N.E. Israeloff and G.B. Alers, J. Magn. Magn. Mater. 114, 87 (1992).

[22] E. Marinari, G. Parisi, J.J. Ruiz-Lorenzo, F. Ritort, Phys. Rev. Lett. 76843 (1996); E. Marinari, G. Parisi, J.J. Ruiz-Lorenzo, in op. cit. [1] pp. 59-98; E. Marinari, G. Parisi, J.J. Ruiz-Lorenzo, On the Phase Structure of the 3D Edwards Anderson spin glass, cond-mat/9802211.

[23] J. Villain, J. Physique France 46, 1843 (1985); for related ideas, see M. Feigel'man, L. Ioffe, Z. Phys. B 51 237 (1983) and M. Gabay, T. Garel, J. Physique 46, 5 (1985).

[24] M. Ocio, J. Hammann and E. Vincent, J. Magn. Magn. Mater. 90-91, 329 (1990). 